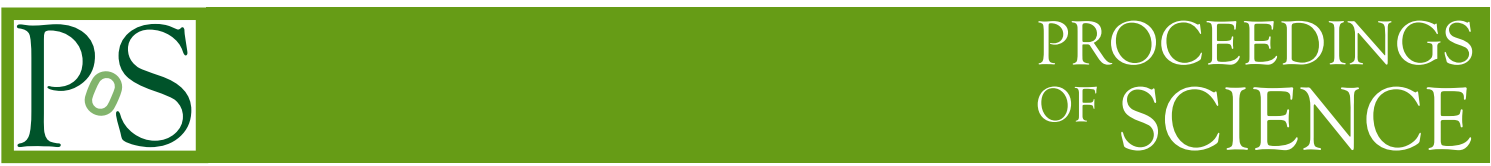

\title{
HI Intensity Mapping with MeerKAT
}

\section{A. Pourtsidou}

Institute of Cosmology \& Gravitation, University of Portsmouth, Dennis Sciama Building, Burnaby Road, Portsmouth, PO1 3FX, United Kingdom

E-mail: alkistis.poursidoulport.ac.uk

Radio detections of the redshifted $21 \mathrm{~cm}$ line with neutral hydrogen (HI) intensity mapping surveys can provide a new way to probe the evolving history of the Universe and tackle fundamental cosmological questions. Intensity mapping (IM) measures the fluctuations of the HI signal tracing the underlying matter distribution, and therefore can be used to reconstruct the matter power spectrum without the need to detect individual galaxies. This means that intensity mapping surveys can observe large volumes very fast, and give us access to large cosmological scales while providing excellent redshift information. We explore the possibility of performing such a survey with the South African MeerKAT radio telescope, which is a precursor to the Square Kilometre Array (SKA). We also propose to use cross-correlations between the MeerKAT intensity mapping survey and optical galaxy surveys, in order to mitigate important systematic effects and produce robust cosmological measurements. Our forecasts show that precise measurements of the HI clustering and lensing signals can be made in the near future. These can be used to constrain $\mathrm{HI}$ and cosmological parameters across a wide range of redshift.

MeerKAT Science: On the Pathway to the SKA,

25-27 May, 2016,

Stellenbosch, South Africa

\footnotetext{
*Speaker.
} 


\section{Introduction}

Intensity mapping $[1,2,3,4,5,6,7,8]$ is an innovative technique that uses neutral hydrogen to map the large-scale structure of the Universe in three dimensions. Intensity mapping surveys use $\mathrm{HI}$ as a tracer of the underlying dark matter distribution but, unlike traditional galaxy surveys, they do not detect individual galaxies; instead, they treat the $21 \mathrm{~cm}$ sky as a diffuse background measuring the integrated intensity of the redshifted $21 \mathrm{~cm}$ line across the sky and along redshift.

Recent studies have shown that we can deliver precision cosmology (e.g. Baryonic Acoustic Oscillations measurements) using SKA Phase 1 and the IM method $[9,10,11]$. Here we will demonstrate that we can use the MeerKAT array to exploit the IM technique and constrain HI and cosmological parameters across a wide range of redshift. This will be an important step towards SKA1_MID large sky surveys, and it will help us maximise their scientific output. We will first investigate what can be achieved using the auto-correlation HI clustering measurements, and then move on to cross-correlations with optical galaxy surveys. Cross-correlations between different surveys are expected to yield precise and robust cosmological constraints. They can alleviate various survey-specific challenges (foregrounds, systematics) that are expected to drop out in cross correlation.

\section{HI intensity mapping with MeerKAT}

MeerKAT $^{1}$ is a 64-dish SKA pathfinder on the planned site of SKA1_MID, with 20 dishes already in place. A large sky intensity mapping survey with MeerKAT has been proposed ${ }^{2}$. It will scan a few thousand degrees on the sky -we take $A_{\text {sky }}=4000 \mathrm{deg}^{2}$ here- in approximately 5 months total observation time. The array will operate in single dish mode in order to access large (cosmological) scales, and there are two frequency bands available ${ }^{3}$ : the $\mathrm{L}$ band $0<z<0.58$ $(900<f<1420 \mathrm{MHz})$, and the UHF band $0.4<z<1.45(580<f<1000 \mathrm{MHz})$. A detailed description of the noise properties of such a survey can be found in [11]. Note that the survey will also take interferometric data simultaneously, and various science cases can be achieved: cosmology, galaxy evolution, clusters, galaxy HI emission, and polarization.

The HI power spectrum can be written as

$$
P^{\mathrm{HI}}(k, z)=\bar{T}_{b}^{2} b_{\mathrm{HI}}^{2} P(k, z),
$$

where $P$ is the matter power spectrum, and $b_{\mathrm{HI}}$ the $\mathrm{HI}$ bias. The mean $21 \mathrm{~cm}$ emission brightness temperature is given by $\bar{T}_{b}(z)=180 \Omega_{\mathrm{HI}}(z) h \frac{(1+z)^{2}}{H(z) / H_{0}} \mathrm{mK}$, where $\Omega_{\mathrm{HI}}$ is the HI density, $H(z)$ the Hubble parameter as a function of redshift $z$, and $H_{0} \equiv 100 h$ its value today [7]. For modelling the $\mathrm{HI}$ density we use $\Omega_{\mathrm{HI}} \simeq 0.00048+0.00039 z$, and for the $\mathrm{HI}$ bias we use $b_{\mathrm{HI}} \simeq 0.67+0.18 z$ [9]. The noise power spectrum $P^{\mathrm{N}}$ is calculated using the formalism described in [11]. In Figure 1 we plot the HI power spectrum and measurements errors for two bins centred at $z=0.3$ and $z=1.2$.

As we can see from the above plots, a very good signal-to-noise ratio can be achieved over a wide range of scales and redshifts. These measurements can be used to constrain HI and cosmological parameters. As a first approach we assume a flat $\Lambda \mathrm{CDM}$ expansion history and keep all

\footnotetext{
${ }^{1} \mathrm{http}: / / w w w . s k a . a c . z a / s c i e n c e-e n g i n e e r i n g / m e e r k a t /$

${ }^{2}$ M. Santos et al, "MeerKLASS: MeerKAT Large Area Synoptic Survey", this volume.

${ }^{3}$ Note that the survey will have to operate in one band only.
} 

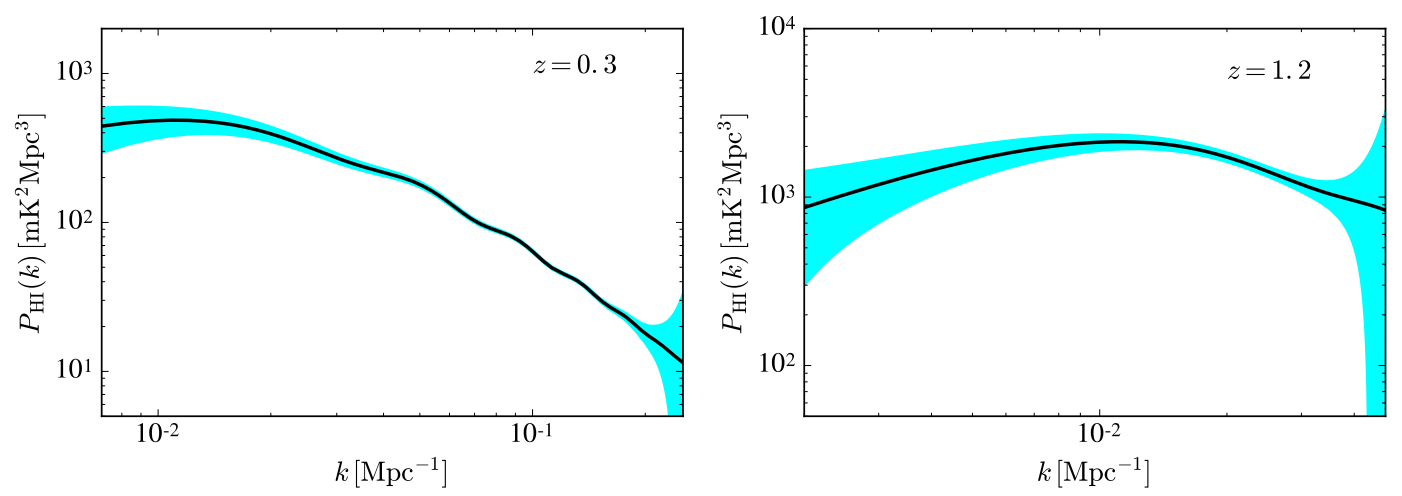

Figure 1: HI detection in autocorrelation with a $4000 \mathrm{deg}^{2}$ survey using MeerKAT. The black solid line is the predicted power spectrum $P_{\mathrm{HI}}(k, z)$ at $z=0.3$ (left) and at $z=1.2$ (right). Note that $z=0.3$ is in the L-band while $z=1.2$ is in the UHF band. The cyan area represents the measurement errors for a total observation time of $\sim 5$ months. The width of the bins is $\Delta z=0.1$ and we have used a $k$-binning $\Delta k=0.01 \mathrm{Mpc}^{-1}$.

cosmological parameters fixed to the Planck 2015 cosmology [12]. Then the only unknown in the HI power spectrum is the prefactor $\Omega_{\mathrm{HI}} b_{\mathrm{HI}}$. We can therefore employ the Fisher matrix formalism [13] to forecast constraints on the HI abundance and bias along redshift. Using the aforementioned MeerKAT IM survey parameters, we find $\delta\left(\Omega_{\mathrm{HI}} b_{\mathrm{HI}}\right) /\left(\Omega_{\mathrm{HI}} b_{\mathrm{HI}}\right)=0.005$ at $z=0.3$ (L band) and $\delta\left(\Omega_{\mathrm{HI}} b_{\mathrm{HI}}\right) /\left(\Omega_{\mathrm{HI}} b_{\mathrm{HI}}\right)=0.03$ at $z=1.2$ (UHF band) [14]. These are more than one order of magnitude better than the currently available constraints from galaxy surveys, intensity mapping, and damped Lyman- $\alpha$ observations (see Table 2 in [15]). In [14] a comprehensive analysis is performed and constraints are derived for multiple redshifts across the L and UHF bands.

Including redshift space distortions (RSD) the HI power spectrum can be written as

$$
P^{\mathrm{HI}}(k, z ; \mu)=\bar{T}_{b}^{2} b_{\mathrm{HI}}^{2}\left[1+\beta_{\mathrm{HI}}(z) \mu^{2}\right]^{2} P(k, z),
$$

where $\mu=\hat{k} \cdot \hat{z}$ and $\beta_{\mathrm{HI}}$ is the redshift space distortion parameter equal to $f / b_{\mathrm{HI}}$ in linear theory, with $f \equiv d \ln D / d \ln a$ the linear growth rate. RSD measurements can be used to break the degeneracy between $\Omega_{\mathrm{HI}}$ and $b_{\mathrm{HI}}[16,14]$. Assuming a $\Lambda \mathrm{CDM}$ expansion history, we find $\delta \Omega_{\mathrm{HI}} / \Omega_{\mathrm{HI}}=0.03$ at $z=0.3$ (L band) and $\delta \Omega_{\mathrm{HI}} / \Omega_{\mathrm{HI}}=0.09$ at $z=1.2$ (UHF band). Another approach is to assume that the mean temperature $\bar{T}_{b}$ has been measured using the smooth part of the HI signal [9], and constrain the $\mathrm{HI}$ bias and various cosmological parameters. Forecasts for constraints on $\left\{f \sigma_{8}, b_{\mathrm{HI}} \sigma_{8}, D_{\mathrm{A}}, H\right\}$ using an IM survey with MeerKAT can be found in [14]. For example, the (marginalised) fractional error on $f \sigma_{8}(z)$ is $3 \%$ at $z=0.3$ (L band) and $13 \%$ at $z=1.2$ (UHF band) for the survey parameters we have considered here.

\section{Cross-correlations with optical galaxy surveys}

Cross correlations between $\mathrm{HI}$ intensity mapping and optical galaxy surveys can provide precise and robust cosmological measurements, as they have the advantage of mitigating major issues like systematics and foreground contaminants that are relevant for one type of survey but not for the other [17, 18]. In [17] the $21 \mathrm{~cm}$ maps acquired at the Green Bank Telescope were combined with 
the WiggleZ galaxy survey [19] to constrain the quantity $\Omega_{\mathrm{HI}} b_{\mathrm{HI}} r$-with $r$ a correlation coefficient accounting for the possible stochasticity in the galaxy and HI tracers- at $z \sim 0.8$ with a statistical fractional error $\sim 16 \%$. The cross correlation power spectrum can be written as [17]

$$
P^{\mathrm{HI}, \mathrm{g}}(k, z)=\bar{T}_{b} b_{\mathrm{HI}} b_{\mathrm{g}} r P(k, z),
$$

with $b_{g}$ the galaxy bias. Here we will consider a forthcoming spectroscopic survey with Euclid-like specifications [20], which is assumed to have a (conservative) $500 \mathrm{deg}^{2}$ sky overlap with MeerKAT, with the observing time reduced accordingly with respect to the full 4,000 $\mathrm{deg}^{2}$ MeerKAT survey. The redshift overlap is $0.7<z<1.4$ (UHF band). In Figure 2 we plot the cross-correlation power spectrum and measurement errors for a bin centred at $z=0.7$. Assuming a $\Lambda$ CDM expansion history and $b_{g}$ measured from the galaxy survey, we find $\delta\left(\Omega_{\mathrm{HI}} b_{\mathrm{HI}} r\right) /\left(\Omega_{\mathrm{HI}} b_{\mathrm{HI}} r\right)=0.06$ at $z=0.7$ and $\delta\left(\Omega_{\mathrm{HI}} b_{\mathrm{HI}} r\right) /\left(\Omega_{\mathrm{HI}} b_{\mathrm{HI}} r\right)=0.14$ at $z=1.2$. Modifying our approach as before by including RSDs and considering $\bar{T}_{b}$ and $b_{\mathrm{g}}$ known we can constrain the growth of structure and expansion history of the Universe. In [14] a comprehensive analysis is performed and constraints are derived for multiple redshifts across the L and UHF bands. Note that assuming an IM survey with SKA1MID and a $7000 \mathrm{deg}^{2}$ overlap with Euclid we find a (marginalised) $4 \%$ error on $f \sigma_{8}$ at $z=0.7$ (see [14] for details). Another possibility is to combine MeerKAT and SKA1-MID IM surveys with photometric galaxy surveys like DES ${ }^{4}$ and $\mathrm{LSST}^{5}$. By doing so we can detect HI clustering and weak gravitational lensing of the $21 \mathrm{~cm}$ emission in cross-correlation [11].

\section{Conclusions}

We have shown that using the MeerKAT pathfinder we can utilise the intensity mapping method to constrain $\mathrm{HI}$ and cosmological parameters across a wide range of redshift. This way we can explore and maximise the scientific output of future large sky surveys with Phase 1 of the SKA. Furthermore, we have proposed to exploit cross-correlations of the $21 \mathrm{~cm}$ intensity maps with galaxies, in order to have the advantage of reducing systematic effects. We believe that the MeerKAT array can be at the forefront of $21 \mathrm{~cm}$ cosmology with the next generation of radio telescopes, and we consider the auto- and cross-correlation approaches highly complementary and synergistic.

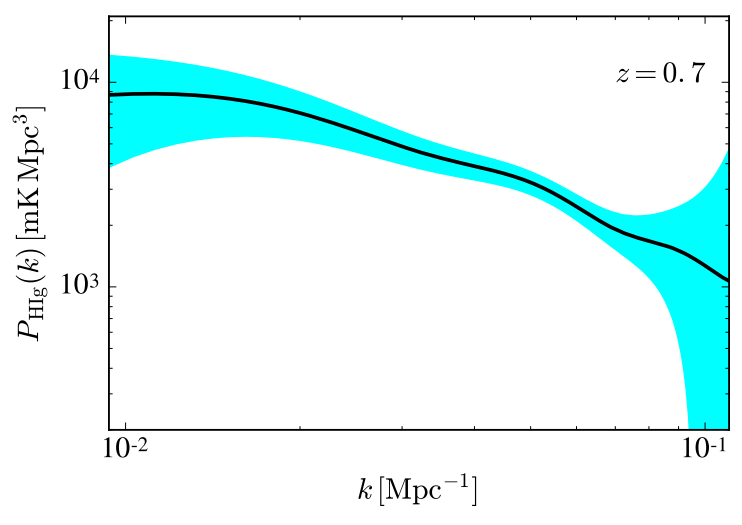

Figure 2: HI detection in cross-correlation assuming the MeerKAT IM survey (UHF band) and a Euclid-like spectroscopic survey with $500 \mathrm{deg}^{2}$ overlap at $z=0.7$. The cyan area represents the measurement errors for a total observation time of $500 \mathrm{hrs}\left(\Delta z=0.1, \Delta k=0.01 \mathrm{Mpc}^{-1}\right)$.

\footnotetext{
${ }^{4}$ https://www.darkenergysurvey.org/

${ }^{5}$ https://www.lsst.org/
} 
Acknowledgments: - I acknowledge support by a Dennis Sciama Fellowship at the University of Portsmouth. I would like to thank David Bacon, Robert Crittenden, Roy Maartens, Ben Metcalf, and Mario Santos for fruitful collaborations and useful discussions.

\section{References}

[1] T.-C. Chang, U.-L. Pen, J. B. Peterson and P. McDonald, Baryon Acoustic Oscillation Intensity Mapping as a Test of Dark Energy, Phys.Rev.Lett. 100 (2008) 091303, [0 709.3672$].$

[2] A. Loeb and S. Wyithe, Precise Measurement of the Cosmological Power Spectrum With a Dedicated 21cm Survey After Reionization, Phys.Rev.Lett. 100 (2008) 161301, [0801.1677].

[3] Y. Mao, M. Tegmark, M. McQuinn, M. Zaldarriaga and O. Zahn, How accurately can $21 \mathrm{~cm}$ tomography constrain cosmology?, Phys.Rev. D78 (2008) 023529, [0802.1710].

[4] J. B. Peterson, R. Aleksan, R. Ansari, K. Bandura, D. Bond et al., 21 cm Intensity Mapping, 0902.3091.

[5] H.-J. Seo, S. Dodelson, J. Marriner, D. Mcginnis, A. Stebbins et al., A ground-based 21cm Baryon acoustic oscillation survey, Astrophys.J. 721 (2010) 164-173, [0 910.5007$].$

[6] R. Ansari, J. Campagne, P. Colom, J. L. Goff, C. Magneville et al., $21 \mathrm{~cm}$ observation of LSS at z 1 Instrument sensitivity and foreground subtraction, Astron.Astrophys. 540 (2012) A129, [1108.1474].

[7] R. Battye, I. Browne, C. Dickinson, G. Heron, B. Maffei et al., HI intensity mapping : a single dish approach, Mon. Not. Roy. Astron. Soc 434 (2013) 1239-1256, [1209. 0343 ].

[8] E. Switzer, K. Masui, K. Bandura, L. M. Calin, T. C. Chang et al., Determination of z 0.8 neutral hydrogen fluctuations using the $21 \mathrm{~cm}$ intensity mapping auto-correlation, Mon.Not.Roy.Astron.Soc. 434 (2013) L46-L50, [1304 . 3712].

[9] P. Bull, P. G. Ferreira, P. Patel and M. G. Santos, Late-time cosmology with $21 \mathrm{~cm}$ intensity mapping experiments, Astrophys.J. 803 (2015) 21, [1 405.1452$].$

[10] M. G. Santos, P. Bull, D. Alonso, S. Camera, P. G. Ferreira et al., Cosmology with a SKA HI intensity mapping survey, 1501.03989.

[11] A. Pourtsidou, D. Bacon, R. Crittenden and R. B. Metcalf, Prospects for clustering and lensing measurements with forthcoming intensity mapping and optical surveys, Mon. Not. Roy. Astron. Soc. 459 (2016) 863-870, [1509.03286].

[12] Planck collaboration, P. A. R. Ade et al., Planck 2015 results. XIII. Cosmological parameters, Astron. Astrophys. 594 (2016) A13, [1502.01589].

[13] M. Tegmark, Measuring cosmological parameters with galaxy surveys, Phys. Rev. Lett. 79 (1997) 3806-3809, [astro-ph/9706198].

[14] A. Pourtsidou, D. Bacon and R. Crittenden, HI and cosmological constraints from intensity mapping, optical, and CMB surveys, Mon. Not. Roy. Astron. Soc. 470 (2017) 4251-4260, [1610 . 04189 ].

[15] H. Padmanabhan, T. R. Choudhury and A. Refregier, Theoretical and observational constraints on the HI intensity power spectrum, Mon. Not. Roy. Astron. Soc. 447 (2015) 3745, [1407. 6366].

[16] K. W. Masui, P. McDonald and U.-L. Pen, Near term measurements with $21 \mathrm{~cm}$ intensity mapping: neutral hydrogen fraction and BAO at $z<2$, Phys. Rev. D81 (2010) 103527, [1 001.4811$].$ 
[17] K. Masui, E. Switzer, N. Banavar, K. Bandura, C. Blake et al., Measurement of 21 cm brightness fluctuations at $z \quad 0.8$ in cross-correlation, Astrophys.J. 763 (2013) L20, [1208 . 0331 ].

[18] L. Wolz et al., Erasing the Milky Way: new cleaning technique applied to GBT intensity mapping data, 1510.05453.

[19] D. Parkinson et al., The WiggleZ Dark Energy Survey: Final data release and cosmological results, Phys. Rev. D86 (2012) 103518, [1210.2130].

[20] L. Amendola et al., Cosmology and Fundamental Physics with the Euclid Satellite, 1606.00180. 